\title{
Dynamic Thiol/Disulphide Homeostasis in Patients with Vaginitis
}

\author{
Raziye Desdicioglu ${ }^{1,}$, , Melahat Yildirim ${ }^{1}$, Betul Ozbek², Gokhan Kılıc ${ }^{1}$, Ozcan Erel², \\ Ayse Filiz Yavuz 1 \\ ${ }^{1}$ Obstetrics and Gynecology Department, Faculty of Medicine, Yildirim Beyazit University, Ankara, Turkey \\ ${ }^{2}$ Biochemistry Department, Faculty of Medicine, Yildirim Beyazit University, Ankara, Turkey
}

\section{Email address:}

raziyedesdicioglu@gmail.com (R. Desdicioglu)

${ }^{*}$ Corresponding author

\section{To cite this article:}

Raziye Desdicioglu, Melahat Yildirim, Betul Ozbek, Gokhan Kılıc, Ozcan Erel, Ayse Filiz Yavuz. Dynamic Thiol/Disulphide Homeostasis in Patients with Vaginitis. Journal of Gynecology and Obstetrics. Vol. 6, No. 1, 2018, pp. 1-5. doi: 10.11648/j.jgo.20180601.11

Received: December 5, 2017; Accepted: December 15, 2017; Published: January 16, 2018

\begin{abstract}
This study investigated the thiol-disulphide balance in vaginitis, which is a local inflammatory condition, through the use of a new method. A cross-sectional study involving 82 women (45 women with vaginitis and 37 women in control group) was conducted at Ataturk Education and Research Hospital, Ankara, Turkey from February 2016 to April 2016. Their

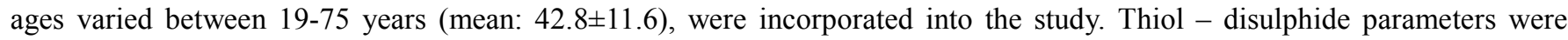
measured and the obtained results were compared. The results showed that the serum disulphide level and disulphide/native thiol ratio in the group with vaginitis $(p=0.03$ and $p=0.04)$ and the native thiol/total thiol ratio in the control group was higher $(p=0.03)$. In this study, it was shown that serum dynamic thiol-disulphide balance in the women with vaginitis had varied. Although vaginitis is a localized infectious inflammatory condition, the increase in the systemic oxidative stress is important. This study is the first article in the literature that investigates the relationship between dynamic thiol-disulphide balance and vaginitis.
\end{abstract}

Keywords: Disulphide, Thiol, Vaginitis

\section{Introduction}

Vaginitis is one of the most common reasons for going to a gynaecology clinic [1]. Vaginitis, a condition characterized by vaginal discharge, itching, irritancy and rash, may have several infectious and non-infectious causes. Recurrent vaginitis may cause social and emotional stress. Normally, a healthy vaginal micro-environment is dependent on the balance between protective microorganisms (lactobacillus) and other pathogenic microorganisms [1]. If this balance is destabilized for any reason in favour of the pathogenic microorganisms, vaginitis can occur. Hydrogen peroxide $\left(\mathrm{H}_{2} \mathrm{O}_{2}\right)$, which is secreted by lactobacilli in the vagina in healthy women, makes vaginal fluids toxic to other microorganisms. It has been shown that there are more $\mathrm{H}_{2} \mathrm{O}_{2}$ producing lactobacilli in the vaginal environment of healthy women than there are in the vaginas of women with bacterial vaginosis [2].
Oxidative stress is a condition that occurs as a result of a disordered balance between reactive oxygen radicals and antioxidant defence mechanisms in the body. The thioldisulphide balance in the human body also plays a role in the antioxidant protection mechanism. The dynamic balance between thiol and disulphide plays a significant role in several vital functions, including programmed cell death (apoptosis), detoxification, antioxidant protection and enzyme activities [3,4]. When this balance shifts towards disulphide, these functions are negatively affected [5]. Studies have shown that thiol-disulphide balance is disrupted in several infectious and inflammatory diseases such as ankylosing spondylitis, inflammatory bowel disease and tonsillopharyngitis [6-8]. An increase in oxidative stress was found in the presence of several local inflammatory diseases, including urinary tract infections, periodontal infections, aphthous stomatitis and acne [9-12].

Though there are no data showing a direct relationship 
between vaginitis and systemic oxidative stress, there are studies showing increased local oxidative stress [1]. There is a clinical place for the use of local antioxidants in treatment of vaginitis to counteract the oxidative stress thought to increase in the vaginal microenvironment. Addition of antioxidants like vitamin $\mathrm{C}$ to local treatments has been shown to increase treatment success [1]. A limited number of papers show that the use of systemic antioxidants in vaginitis situations is superior to placebo [13].

The aim of this study to examine dynamic thiol/disulfide homeostatic status in women with vaginitis by using a new and automated analysis method [5]. No studies were found that examine the relationship between serum thiol/disulphide balance and vaginitis by using this new method.

\section{Methods}

A cross-sectional study involving 82 women (45 women with vaginitis and 37 women in control group) was conducted at Ataturk Education and Research Hospital, Ankara, Turkey from February 2016 to April 2016. The study protocol was approved by the ethical committee of Ankara Ataturk Training and Research Hospital. Written and signed informed consent was obtained from all study participants.

Of the women with vaginitis included in study, 19 had candida vaginitis, 15 had bacterial vaginosis and 11 had trichomonas vaginitis. The diagnosis of vaginitis was confirmed by laboratory tests after suggestion by the symptoms of the patients (vaginal discharge, itching, unpleasant odour, etc.) as well as examination findings. Amsel criteria were used for the diagnosis of bacterial vaginosis [14]. The Amsel criteria are thick, homogenous vaginal discharge and $\mathrm{pH}$ of the vagina $>4.5$, positive whiff test and more than $20 \%$ clue cells observed on wet preparations. Patients meeting at least three of these criteria were diagnosed with bacterial vaginosis. Trichomonas diagnosis was made according to the patients complaints and examination findings, in addition to observation of moving parasites on wet preparations. Patients considered to have vaginal candidiasis due to typical complaints and examination findings had definite diagnosis confirmed via a vaginal discharge sample mixed with $10 \%$ potassium hydroxide $(\mathrm{KOH})$ and examined by microscope with observation of fungal hyphae. This study included women with symptoms of acute vaginitis. Women with chronic or recurrent vaginitis symptoms were excluded. Pregnant women, those diagnosed with a sexually transmitted disease (e.g. HPV positive), those with chronic systemic disease, and those with acute infections were excluded from the study. To eliminate urinary tract infection (UTI), the patients were asked to provide urine samples, and those with UTI were excluded from the study. The ages, body mass indexes (BMI) and gynaecological and obstetric histories of the patients and whether or not they used a vaginal douching method were all recorded. Serum thiol-disulphide homeostasis was determined with a recently developed novel and automatic measurement method by using an automated clinical chemistry analyser (Roche, Cobas 501, Mannheim, Germany) [5].

The compliance of the variables with normal distribution was evaluated via the Kolmogorov-Smirnov test. For the evaluation of inter-group differences, student's $t$-test was used. The value $p<0.05$ was accepted as the statistical significance limit.

\section{Results}

There was no difference observed between the two groups in the study in terms of age, BMI, gravida, parity, menopausal situation and vaginal douche history $(p>0.05)$.

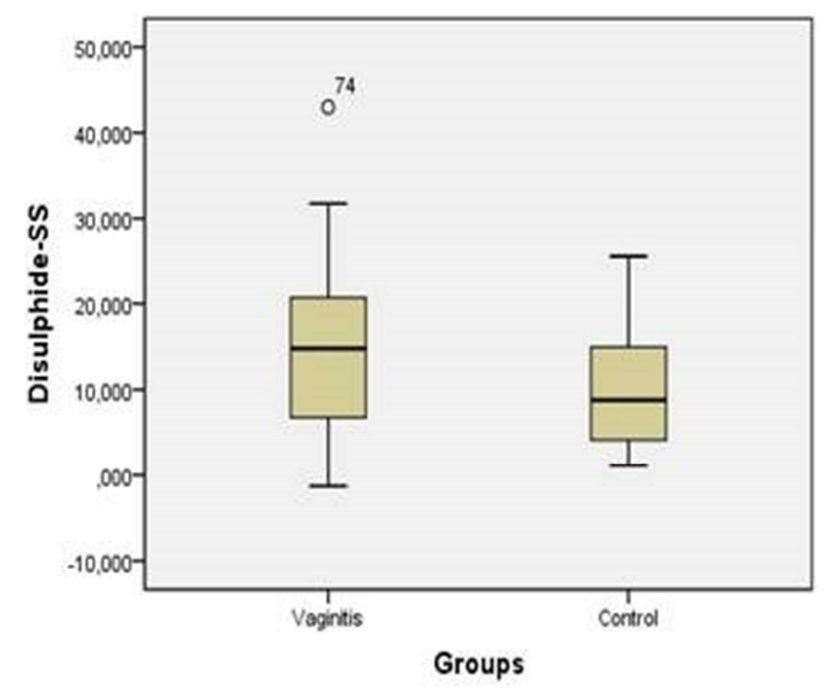

Figure 1. Comparison of disulphide levels in the two groups.

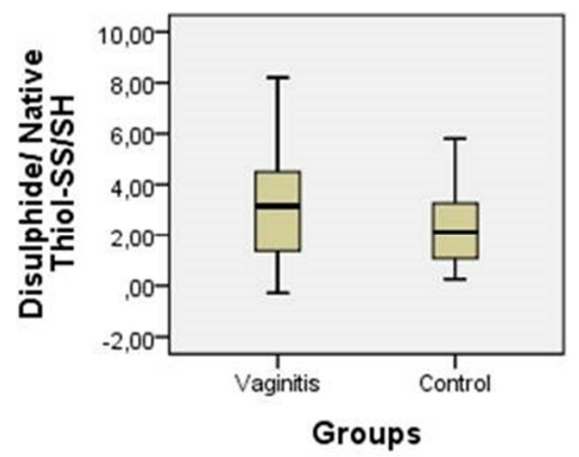

Figure 2. Disulphide/native thiol ratio between the two groups.

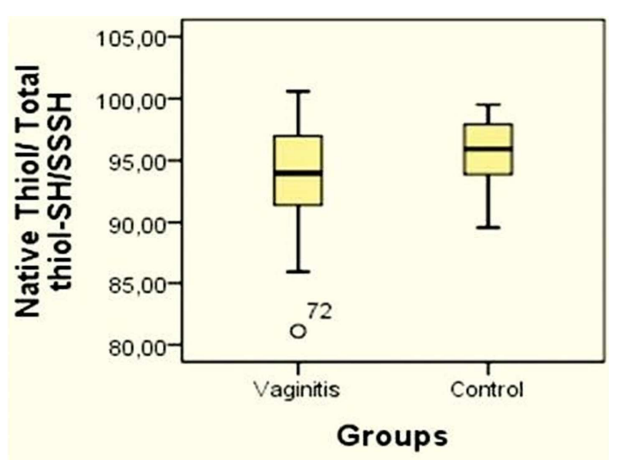

Figure 3. Native thiol/total thiol ratio between the two groups. 
The demographic data pertaining to the patients are summarized in Table 1. The C-reactive protein level (CRP) between the vaginitis and control groups was not different. The native thiol levels did not differ between the two groups, whereas the disulphide level proved to be significantly higher in the vaginitis group $(p<0.05)$ (Figure 1). While the disulphide/native thiol ratio proved to be significantly high in the group with vaginitis, the native thiol/total thiol ratios in the control group were found to be higher $(p<0.05)$ (Figure. 2 and 3). Even though the disulphide/total thiol ratio in the group with vaginitis was high, no statistically significant difference was observed $(p>0.05)$ from the ratio in the control group. The total serum thiol level in the group with vaginitis and in the control group was found to be different at the limit $(p=0.05)$. The results are summarized in Table 2 .

Table 1. Demographic characteristics of the groups.

\begin{tabular}{llll}
\hline Parameters & Vajinitis group $(\mathbf{n}=\mathbf{4 7})$ & Control group $(\mathbf{n = 3 5})$ & $\boldsymbol{P}$ \\
\hline Age $($ years $)$ & $40.8 \pm 10.4$ & $45.3 \pm 12.6$ & $p>0.05$ \\
BMI $\left(\mathrm{kg} / \mathrm{m}^{2}\right)$ & $29.3 \pm 5.1$ & $30.3 \pm 6.2$ & $p>0.05$ \\
Gravida $(\mathrm{n})$ & $3.8 \pm 2.7$ & $2.9 \pm 2.2$ & $p>0.05$ \\
\hline
\end{tabular}

Table 2. Laboratory parameters of the groups.

\begin{tabular}{llll}
\hline Parameters & Vaginitis group $(\mathbf{n}=\mathbf{4 7})$ & Control group $(\mathbf{n}=\mathbf{3 5})$ & $\boldsymbol{p}$ value* \\
\hline Native thiol $(\mu \mathrm{mol} / \mathrm{L})$ & $470,94 \pm 33,42$ & $460,69 \pm 54,93$ \\
Total thiol $(\mu \mathrm{mol} / \mathrm{L})$ & $502,26 \pm 36,56$ & $481,83 \pm 55,81$ \\
Disulphide $(\mu \mathrm{mol} / \mathrm{L})$ & $14,65 \pm 9,73$ & $10,46 \pm 6,91$ & 0.3 \\
\% Disulphide/ native thiol & $3,14 \pm 2,07$ & $2,31 \pm 1,54$ & 0.05 \\
\% Disulphide/total thiol & $2,88 \pm 1,82$ & $2,17 \pm 1,39$ & $0.03 *$ \\
\% Native /total thiol & $93,86 \pm 4,09$ & $95,60 \pm 2,76$ & $0.04 *$ \\
C-Reactive protein $(\mathrm{mg} / \mathrm{L})$ & $6.34 \pm 6.07$ & $6.00 \pm 5.12$ & 0.05 \\
\hline
\end{tabular}

*; $\mathrm{p}<0.05$ is statistically significant

\section{Discussion}

The present study explored whether the systemic thiol/disulphide balance was different in women with vaginitis compared to women without vaginitis. To our knowledge, this is the first study to assess the relationship between vaginitis and thiol metabolism. Herein it was demonstrated that there was an increase in disulphide levels indicative of oxidative stress in the systemic circulation system of women with vaginitis, a local infection. Again, the disulphide rate in the group with vaginitis was determined to be higher when compared with the control group. The native thiol/total thiol ratios, on the other hand, proved to be higher in the control group.

The relationship between oxidative stress and vaginitis has been evaluated in previous studies. Guo et al., in their study conducted in 2013, evaluated malondialdehyde (MDA), catalase (CAT), superoxide dismutase (SOD) and $\mathrm{H}_{2} \mathrm{O}_{2}$ levels in cervicovaginal fluid.

In that study, it was found that MDA and $\mathrm{H}_{2} \mathrm{O}_{2}$ levels were high in the vaginal fluids of the patients with vaginitis, whereas the CAT levels were low. While MDA is a lipid peroxidation product indicating oxidative stress, CAT and SOD are found in the group of antioxidant enzymes [15]. Although $\mathrm{H}_{2} \mathrm{O}_{2}$ is a protective molecule against other microorganisms when it is released at low levels by lactobacilli, higher amounts of this molecule cause oxidative damage [1].

In a study in which apoptosis and oxidative stress in nonspecific vaginitis (bacterial vaginosis, NSV) were investigated, it was determined that vaginal oxidative stress products (MDA and $\mathrm{H}_{2} \mathrm{O}_{2}$ ) increased in patients with bacterial vaginosis, whereas the antioxidant enzyme levels (SOD, CAT) decreased. The same study showed this situation to be inverted after local treatment. In that study, local examination was performed on the vaginal discharge fluid [1]. In the study, however, a systemic evaluation was performed, and oxidative stress was shown to have increased as evidenced by serum measurements from all patients with vaginitis. All types of vaginitis were evaluated together in this study, whereas in the previous study, only patients with bacterial vaginosis were incorporated in the study.

In an experimental study in which the relationship between oxidative stress markers and recurrent vulvovaginal candidiasis in serum and vaginal tissue was investigated, it was shown that serum MDA and glutathione levels in diabetic rats with recurrent candidal vaginitis were high [16]. It was observed after treatment of diabetes and/or candida that serum oxidative stress markers decreased [16]. In a study conducted on cells isolated from the vaginal discharge of patients with recurrent vulvovaginal candidiasis (RVVC), it was demonstrated that the candida albicans strains isolated from these patients had higher levels of reduced thiol. It was considered that they were protected from oxidative stress and became resistant to treatment and thus experienced recurrent infections through this mechanism [17]. In another study in which oxidative stress was evaluated in the amniotic fluid of pregnant women with bacterial vaginosis, it was stated that MDA, SOD and GSh-PX levels did not show any significant difference between the control and patient groups [18].

Studies have investigated the relationship between thiols and local and systemic diseases. It was shown that serum disulphide levels and disulphide/native thiol ratios increased in children with viral tonsillopharyngitis [19]. It was also demonstrated that disulphide levels were increased in the 
presence of some degenerative diseases, in smokers, those with diabetes, obese patients, those with inflammatory bowel diseases, in AIDS patients and in pregnant women with familial Mediterranean fever (FMF) [5, 7, 20, 21].

In this study, the serum disulphide level and disulphide/native thiol ratios proved to be significantly high in the group with vaginitis, whereas the native thiol/total thiol ratio in the control group was significantly high. In this study it was ascertained that native thiol levels, though insignificant, proved to be lower in the patient population. In the previously conducted studies, it was shown that native thiol level decreased in the presence of infections such as brucellosis as well as Crimean-Congo haemorrhagic fever and viral and bacterial tonsillopharyngitis [8, 22, 23]. The results of present study are in agreement with the studies in the literature.

The relationship between locally increased oxidative stress and vaginitis allows local treatments involving antioxidants (e.g. vitamin C) to be used to treat vaginitis. In a cell-culture study conducted on this subject, the use of vitamin $\mathrm{C}$ in addition to metronidazole treatment was shown to significantly decrease oxidative stress markers [1]. In that study, not only was vitamin $\mathrm{C}$ treatment administered but it was also used along with metronidazole. In another study, it was shown that vaginal vitamin $\mathrm{C}$ preparations in patients with non-specific vaginitis increased the acidity of the vagina and ensured an increase in treatment efficiency and in the lactobacilli rate in the vagina [13]. In light of present study indicating that systemic oxidative stress increases in vaginitis, we are of the opinion that the systemic use of medications containing antioxidant molecules (vitamin $\mathrm{C}, \mathrm{E}$ alpha lipoic acid, $\mathrm{N}$-acetylcysteine, etc.) could be used in the treatment of vaginitis and in the prevention of its recurrence.

Limitations of this study include the comparatively small number of patients and that thiol levels were only studied in serum while local fluid was not evaluated. In addition, preand post-treatment levels were not compared, oxidative stress and antioxidant markers (MDA, SOD, GSH-Px), except for thiol, were not studied and the agents of vaginitis were subject to a total evaluation without forming separate groups.

The powerful aspect of present study, however, is the fact that this is the first study that investigates the relationship between dynamic serum thiol balance and vaginitis. All patients with infectious and inflammatory diseases, as well as those with additional chronic diseases and those who used any type of medication, were excluded from the study.

\section{Conclusion}

In conclusion, it was determined that the dynamic serum thiol disulphide balance shifted in the direction of disulphide (towards the oxidized side) in the case of vaginitis. These findings suggested that oxidative stress may play a role in the pathogenesis of vaginitis. This outcome is of importance in terms of indicating that systemic oxidative stress increases during local inflammatory processes. However, larger studies are needed to support our findings.
The authors declare that they have no any conflict of interest.

\section{Abbreviations}

\author{
$\mathrm{H}_{2} \mathrm{O}_{2}$ : Hydrogen peroxide \\ HPV: Human papilloma virus \\ UTI: Urinary tract infection \\ BMI: Body mass index \\ CRP: C-reactive protein \\ Cys: Cysteine \\ MDA: Malondialdehyde \\ CAT: Catalase \\ SOD: Superoxide dismutase \\ GSh-PX: Glutathione peroxidase \\ RVVC: Recurrent vulvovaginal candidiasis \\ FMF: Familial Mediterranean fever
}

\section{References}

[1] Chen Z, Zhang Z, Zhang H, Xie B. 2015 Analysis of the Oxidative Stress Status in Nonspecific Vaginitis and Its Role in Vaginal Epithelial Cells Apoptosis. BioMed research international. 2015; http://dx.doi.org/10.1155/2015/795656.

[2] Eschenbach DA, Davick PR, Williams BL, Klebanoff SJ, YoungSmith K, Critchlow CM, et al. Prevalence of hydrogen peroxideproducing Lactobacillus species in normal women and women with bacterial vaginosis. J Clin Microbiol 1989; 27 (2): 251-256.

[3] Biswas S, Chida AS, Rahman I. Redox modifications of protein-thiols: emerging roles in cell signaling. Biochem Pharmacol 2006; 71 (5): 551-564.

[4] Circu M, Aw TY. Reactive oxygen species, cellular redox systems, and apoptosis. Free Radic Biol Med 2010; 48 (6): 749-762.

[5] Erel O, Neselioglu S. A novel and automated assay for thiol/disulphide homeostasis. Clin Biochem 2014; 47 (18): 326-332.

[6] Dogru A, Balkarli A, Cetin GY, Neselioglu S, Erel O, Tunc SE. et al. 2016. Thiol/disulphide homeostasis in patients with ankylosing spondylitis. Bosnian Journal of Basic Medical Sciences 2016; Doi: http://dx.doi.org/10.17305/bjbms.2016.1001

[7] Yuksel M, Ates I, Kaplan M, Alisik M, Erel O, Saygili F et al. The dynamic thiol/disulphide homeostasis in inflammatory bowel disease and its relation with disease activity and pathogenesis. Int J Colorectal Dis 2016; 31 (6): 1229-1231.

[8] Kara SS, Erel O, Demirdag TB, Cura BC, Gulhan B, Neselioglu $\mathrm{S}$ et al. Alteration of thiol-disulphide homeostasis in acute tonsillopharyngitis. Redox Report 2016; DOI: 10.1080/13510002.2016.1173328

[9] Ciragil P, Kurutas EB, Gul M, Kilinc M, Aral M, Guven A. The effects of oxidative stress in urinary tract infection during pregnancy. Mediators of inflammation 2005; 5: 309-311.

[10] Dursun E, Akaln FA, Genc T, Cinar N, Erel O, Yildiz BO. Oxidative Stress and Periodontal Disease in Obesity. Medicine 2016; 95 (12): e3136. 
[11] Cimen MYB, Kaya TI, Eskandari G, Tursen U, Ikizoglu G, Atik U. Oxidant/antioxidant status in patients with recurrent aphthous stomatitis. Clin Exp Dermatol 2003; 28 (6): 647650 .

[12] Perihan O, Ergul KB, Neslihan D, Filiz A. The activity of adenosine deaminase and oxidative stress biomarkers in scraping samples of acne lesions. Journal of Cosmetic Dermatology 2012; 11 (4): 323-28.

[13] Petersen EE, Magnani P. Efficacy and safety of Vitamin C vaginal tablets in the treatment of non-specific vaginitis: a randomised, double blind, placebo-controlled study. Eur J Obstet Gynecol Reprod Biol 2004; 117 (1): 70-75.

[14] Hainer BL, Gibson MV. Vaginitis: Diagnosis and Treatment. Am Fam Physician 2011; 83: 7.

[15] Guo HY, Hu XM, Han DD, Wang ZP, Meng L. Lipid peroxidation and antioxidant status in vagina microenvironment of patients with several common vaginitis. Clin Exp Obstet Gynecol 2012; 40 (3): 331-336.

[16] Kustimur S, Kalkanci A, Akbulut G, Gonul B, Bulduk E, Aksakal FN. et al. The effect of vaginal candidiasis on the levels of the oxidative biomarkers in plasma and tissue samples of diabetic rats. Mycopathologia 2007; 164 (5): 217 224.

[17] Ratti BA, Godoy JSR, Mendonca PSB, Bidoia DL, Nakamura TU, Consolaro MEL, et al. Microbicidal activity of neutrophils is inhibited by isolates from recurrent vaginal candidiasis (RVVC) caused by Candida albicans through fungal thioredoxin reductase. Cell. Immunol 2015; 293 (1): 22-29.

[18] Bogavac M, Lakic N, Simin N, Nikolic A, Sudji J, Bozin B. Bacterial vaginosis and biomarkers of oxidative stress in amniotic fluid. The Journal of Maternal-Fetal \& Neonatal Medicine 2012; 25 (7): 1050-1054.

[19] Eren Y, Dirik E, Neşelioglu S, Erel O. 2015. Oxidative stress and decreased thiol level in patients with migraine: crosssectional study. Acta Neurol Belg 2015; 115 (4): 643-649.

[20] Walmsley SL, Winn LM, Harrison ML, Uetrecht JP, Wells PG. Oxidative stress and thiol depletion in plasma and peripheral blood lymphocytes from HIV-infected patients: toxicological and pathological implications. AIDS 1997; 11 (14): 16891697.

[21] Yucel A, Sanhal CY, Daglar K, Kara O, Uygur D, Erel O. Thiol/disulphide homeostasis in pregnant women with Familial Mediterranean fever. Redox Report 2016; Doi: $10.1080 / 13510002.2016 .1168590$

[22] Esen R, Aslan M, Kucukoglu ME, C1kman A, Yakan U, Sunnetcioglu M, et al. 2015. Serum paraoxonase activity, total thiols levels, and oxidative status in patients with acute brucellosis. Wien Klin Wochenschr 2015; 127 (11-12): 427433.

[23] Karadag-Oncel E, Erel O. Ozsurekci Y, Caglayık-Yagci D. Kaya A. Gozel MG, et al. Plasma oxidative stress and total thiol levels in crimean-congo hemorrhagic Fever. Japanese journal of infectious diseases 2014; 67 (1): 22-26. 